\title{
A Closed-Form Solution for Robust Portfolio Selection with Worst-Case CVaR Risk Measure
}

\author{
Le Tang ${ }^{1}$ and Aifan Ling ${ }^{2}$ \\ ${ }^{1}$ School of Foreign Languages and International Business, Jiangxi University of Technology, Nanchang 330098, China \\ ${ }^{2}$ School of Finance, Jiangxi University of Finance and Economics, Nanchang 330013, China
}

Correspondence should be addressed to Aifan Ling; aiffling@163.com

Received 24 March 2014; Accepted 11 June 2014; Published 2 July 2014

Academic Editor: Guangchen Wang

Copyright (C) 2014 L. Tang and A. Ling. This is an open access article distributed under the Creative Commons Attribution License, which permits unrestricted use, distribution, and reproduction in any medium, provided the original work is properly cited.

With the uncertainty probability distribution, we establish the worst-case CVaR (WCCVaR) risk measure and discuss a robust portfolio selection problem with WCCVaR constraint. The explicit solution, instead of numerical solution, is found and two-fund separation is proved. The comparison of efficient frontier with mean-variance model is discussed and finally we give numerical comparison with VaR model and equally weighted strategy. The numerical findings indicate that the proposed WCCVaR model has relatively smaller risk and greater return and relatively higher accumulative wealth than VaR model and equally weighted strategy.

\section{Introduction}

$\mathrm{VaR}$ (value at risk) has been a popular risk measure in finance industry and academic research and is written in New Basel Accord. But two difficulties are faced by user: (1) the explicit expression of VaR is unavailable unless the normal distribution assumption is done and (2) VaR, as a risk measure tool, does not satisfy the coherent axiom [1]. Hence, an approximation of $\mathrm{VaR}$ is often considered in practice by either assuming normal distribution or simulation method based historical data. Rockafellar and Uryasev [2] proposed an alternative risk measure, namely, conditional VaR (CVaR), which is coherent and provided a linear programming approximate with historical data. But the assumption that the return of risky asset follows the normal distribution is usually done when one computes $\mathrm{CVaR}$ by parameterized approaches. As we know, normal distribution can usually underestimate the loss of the rare event and is not clearly a very good approximation of the return of risky asset. This is still a challenge for computing an explicit expression of CVaR without any special distribution information. The current paper will explore this problem and establish the mean-CVaR portfolio model without probability distribution assumption.

Robust portfolio problems with parameters uncertainty are recently paid close attention to. Goldfarb and Iyengar [3], for instance, considered a class of robust portfolio problem with risk factors in which they solve numerically robust mean-variance portfolio problem, robust downside risk portfolio problem with normal distribution, and robust Sharpe ratio portfolio problem; see also, Costa and Paiva [4], Halldórsson and Tütüncü [5], Tütüncü and Koenig [6], Lu [7], and Ling and Xu [8] for the relative researches. The uncertainty of models above is only from the parameters under the deterministic distribution and cannot capture the uncertainty in distribution. El Ghaoui et al. [9] proposed the worstcase VaR (WCVaR) risk measure and considered a portfolio selection problem with minimization of WCVaR. Zhu and Fukushima [10] proposed the worst-case CVaR risk measure and discussed a robust mean-CVaR portfolio model with uncertainty discrete distribution. Some similar researches can be found in Zhu et al. [11] and Huang et al. [12]. A richer literature can be referred to in Fabozzi et al. [13].

We define the worst-case CVaR with uncertainty distribution including the continuous and discrete distribution and consider a portfolio selection problem with WCCVaR as risk measure. Our results extend that of Zhu and Fukushima [10] for which they considered only the discrete case to the case including the continuous and discrete distribution. Most of methods for robust portfolio problems are that one converts first the problems into convex cone (e.g., linear 
programming, second-order cone programming, or positive semidefinite programming) and then solves them numerically. Differently from these numerical methods, we consider an analytic solution approach for the proposed robust meanWCCVaR problem. We discuss two cases of the proposed robust problems with and without risky-free asset and prove two-fund separable theorem. Numerical results and comparisons with VaR and equally weighted strategy for real market data are reported.

The outline of this paper is arranged as follows. We introduce the definition of worst-case CVaR, establish meanWCCVaR portfolio model, and give the closed-form solution in Section 2 and Section 3 proves the two-fund separation theorem. The extension of the model with risky-free asset is considered in Section 4. Numerical results are reported in Section 5 .

\section{Mean-WCCVaR Portfolio Model}

We consider mainly an investing and holding strategy in this paper for which the investor allocates his (her) assets at time 0 and collects his (her) returns of portfolio at time 1. Generally speaking, two things must be done at time 0: one is that the investor needs to estimate the returns of risky assets at time 1 using the available information at time 0 and another is that the investor must choose an optimal decision to allocate his (her) wealth.

Let there be $n$ available risky assets in the market and let their random returns vector be denoted by $\mathbf{r}=\left(r_{1}, \ldots, r_{n}\right)^{T} \in$ $\mathbb{R}^{n}$. The expected returns and covariance matrix are denoted, respectively, by $\boldsymbol{\mu}=\mathbb{E}[\mathbf{r}]=\left(\mu_{1}, \ldots, \mu_{n}\right)^{T} \in \mathbb{R}^{n}$ and $\Sigma=$ $\left(\sigma_{i j}\right)_{n \times n}$, where $\sigma_{i i}=\sigma_{i}^{2}=\operatorname{var}\left(r_{i}\right)$ is variance of asset $i(i=$ $1, \ldots, n)$. The rate of return of risky-free asset is denoted by $r_{f}$. The portfolio vector is $\mathbf{w}=\left(w_{1}, \ldots, w_{n}\right)^{T} \in \mathbb{R}^{n}$ with $w_{i}$ the proportion of wealth invested in asset $i$. The weight of wealth invested in risky-free asset is denoted by $w_{f}$. Let $f(\mathbf{w}, \mathbf{r})$ be the loss for portfolio vector $\mathbf{w}$ and satisfy $\mathbb{E}[|f(\mathbf{w}, \mathbf{r})|]<+\infty$, and let $F(\cdot)$ be the joint cumulative probability distribution of random vector $\mathbf{r}$. Then the probability that the loss $f(\mathbf{w}, \mathbf{r})$ is not greater than a given constant $\alpha$ is

$$
\Psi(\mathbf{w}, \alpha)=\mathbb{P}\{f(\mathbf{w}, \mathbf{r}) \leq \alpha\}=\int_{f(\mathbf{w}, \mathbf{r}) \leq \alpha} d F(\mathbf{r}) .
$$

Let $\beta \in(0,1)$; then, with confidence level $\beta, \operatorname{VaR}_{\beta}$ can be expressed as

$$
\operatorname{VaR}_{\beta}(\mathbf{w})=\min \{\alpha \in \mathbb{R} \mid \Psi(\mathbf{w}, \alpha) \geq \beta\}
$$

Rockafellar and Uryasev [2] defined CVaR as the conditional expectation of loss $f(\mathbf{w}, \mathbf{r})$ greater than $\operatorname{VaR}_{\beta}$. With the definition of CVaR and the given confidence level $\beta$, the mathematic formulation of CVaR can be written as

$$
\begin{aligned}
\operatorname{CVaR}_{\beta}(\mathbf{w}) & =\mathbb{E}\left[f(\mathbf{w}, \mathbf{r}) \mid f(\mathbf{w}, \mathbf{r}) \geq \operatorname{VaR}_{\beta}(\mathbf{w})\right] \\
& =\frac{1}{1-\beta} \int_{f(\mathbf{w}, \mathbf{r}) \geq \operatorname{VaR}_{\beta}(\mathbf{w})} f(\mathbf{w}, \mathbf{r}) d F(\mathbf{r}) .
\end{aligned}
$$

Let

$$
H_{\beta}(\mathbf{w}, \alpha)=\alpha+\frac{1}{1-\beta} \mathbb{E}\left[(f(\mathbf{w}, \mathbf{r})-\alpha)_{+}\right] .
$$

Then $\mathrm{CVaR}_{\beta}(\mathbf{w})$ can be expressed further as [2]

$$
\mathrm{CVaR}_{\beta}(\mathbf{w})=\min _{\alpha} H_{\beta}(\mathbf{w}, \alpha),
$$

where

$$
(a)_{+}= \begin{cases}a, & a>0 \\ 0, & a \leq 0 .\end{cases}
$$

Clearly, it is not possible to get an exact result of CVaR by (4) if we have not any information on the distribution of random vector $\mathbf{r}$. Some sampling or simulation methods are used to computes the approximation of $\mathrm{CVaR}$ in the literature. We explore a closed-form solution in this paper with only partial distribution assumptions for random vector $\mathbf{r}$, for which we assume that random vector $\mathbf{r}$ follows a family of distributions $\mathscr{D}$ defined by

$$
\mathscr{D}=\{\mathbf{r} \mid \mathbb{E}[\mathbf{r}]=\boldsymbol{\mu}, \operatorname{Cov}(\mathbf{r})=\Sigma>0\},
$$

where $\Sigma>0$ means that $\Sigma$ is a positive definite matrix and $\mathscr{D}$ is called the uncertainty set of distribution of random vector $\mathbf{r}$. Clearly, $\mathscr{D}$ is a distribution family with given mean value $\boldsymbol{\mu}$ and covariance matrix Cov, where $\boldsymbol{\mu}$, Cov are assumed to be known. We then can compute $\mathrm{CVaR}_{\beta}$ when the worst-case probability distribution in $\mathscr{D}$ occurs. To this end, we define worst-case CVaR as follows.

Definition 1. Let $\beta \in(0,1)$, worst-case CVaR (WCCVaR) of portfolio w under uncertainty set $\mathscr{D}$ is defined by

$$
\operatorname{WCCVaR}_{\beta}(\mathbf{w})=\sup _{F \in \mathscr{D}} \operatorname{CVaR}_{\beta}(\mathbf{w})=\sup _{F \in \mathscr{D}} \min _{\alpha} H_{\beta}(\mathbf{w}, \alpha) .
$$

Noticing that $H_{\beta}(\mathbf{w}, \alpha)$ is the convex function of $\alpha$ [14], then we have from max-min theorem [15]

$$
\mathrm{WCCVaR}_{\beta}(\mathbf{w})=\sup _{F \in \mathscr{D}} \min _{\alpha} H_{\beta}(\mathbf{w}, \alpha)=\min _{\alpha} \sup _{F \in \mathscr{D}} H_{\beta}(\mathbf{w}, \alpha) .
$$

The following results are straightforward and a similar proof can be found in [10].

Theorem 2. $\mathrm{WCCVaR}_{\beta}(\mathbf{w})$ is a coherent risk measure and satisfies that

$$
\operatorname{WCCVaR}_{\beta}(\mathbf{w}) \geq \operatorname{CVaR}_{\beta}(\mathbf{w}) \geq \operatorname{VaR}_{\beta}(\mathbf{w}) .
$$

Hence, $\mathrm{WCCVaR}_{\beta}(\mathbf{w})$ can be used as a risk measure and if the investor measures the risk of portfolio based on WCC$\mathrm{VaR}$, then we can establish the mean-WCCVaR portfolio model by

$$
\begin{array}{ll}
\underset{\mathbf{w}}{\max } & \boldsymbol{\mu}^{T} \mathbf{w} \\
\text { s.t. } & \operatorname{WCCVaR}_{\beta}(\mathbf{w}) \leq \tau, \\
& \mathbf{e}^{T} \mathbf{w}=1,
\end{array}
$$


where $\tau>0$ is a preset constant. We solve mainly problem (RP1) by exploring an explicit approach. To this end, for convenience, we denote sometime $\mathbf{r} \in \mathscr{D}$ by $\mathbf{r} \sim(\boldsymbol{\mu}, \Sigma)$. The following result is helpful for our analysis later.

Lemma 3 (see [15]). Let $\xi$ be a random variable with mean value $\mu$ and variance $\sigma . \rho$ is any real number. Then, for the supper bound of $\mathbb{E}\left[(\rho-\xi)_{+}\right]$, we have

$$
\sup _{\xi \sim(\mu, \sigma)} \mathbb{E}\left[(\rho-\xi)_{+}\right]=\frac{\rho-\mu+\sqrt{\sigma^{2}+(\rho-\mu)^{2}}}{2} .
$$

Lemma 4 (see $[14,16])$. Let for any vector $\mathbf{a} \in \mathbb{R}^{n}$,

$$
\begin{aligned}
& S_{1}=\left\{\mathbf{a}^{T} \mathbf{r} \mid \mathbf{r} \in \mathscr{D}\right\}, \\
& S_{2}=\left\{\eta \mid \mathbb{E}(\eta)=\mathbf{a}^{T} \boldsymbol{\mu}, \operatorname{Var}(\eta)=\mathbf{a}^{T} \sum \mathbf{a}\right\} .
\end{aligned}
$$

Then $S_{1}=S_{2}$.

Lemma 3 provides an upper bound of 1-order lower partial moment for one dimensional random variable and Lemma 4 provides a relationship of uncertainty set between one dimensional and several dimensional random variable with given mean value and variance. Lemma 4 indicates also that $S_{1}$ (or $S_{2}$ ) is in fact a single variable distribution family with given mean $\mathbf{a}^{T} \boldsymbol{\mu}$ and variance $\mathbf{a}^{T} \sum \mathbf{a}$, where $\mathbf{a}, \boldsymbol{\mu}$, and $\boldsymbol{\Sigma}$ are known. Further, we can obtain an explicit expression for sup in (4) if the loss $f(\mathbf{w}, \mathbf{r})$ is linear.

Lemma 5. If $f(\mathbf{w}, \mathbf{r})=-\mathbf{r}^{T} \mathbf{w}$, then for any $\mathbf{r} \in \mathscr{D}$ and $\alpha \in \mathbb{R}$, we have

$$
\begin{array}{rl}
\sup _{\mathbf{r} \in \mathscr{D}} & \mathbb{E}\left[(f(\mathbf{w}, \mathbf{r})-\alpha)_{+}\right] \\
= & \sup _{\mathbf{r} \in \mathscr{D}} \mathbb{E}\left[\left(-\alpha-\mathbf{r}^{T} \mathbf{w}\right)_{+}\right] \\
& =\frac{1}{2}\left(\sqrt{\mathbf{w}^{T} \sum \mathbf{w}+\left(\boldsymbol{\mu}^{T} \mathbf{w}+\alpha\right)^{2}}-\left(\boldsymbol{\mu}^{T} \mathbf{w}+\alpha\right)\right) .
\end{array}
$$

Proof. Let $\mathbf{a}=\mathbf{w}$ and $\eta=\mathbf{w}^{T} \mathbf{r}$. Then, it follows from Lemma 4 that

$$
\begin{array}{rl}
\sup _{\mathbf{r} \in \mathscr{D}} & \mathbb{E}\left[(f(\mathbf{w}, \mathbf{r})-\alpha)_{+}\right] \\
= & \sup _{\mathbf{r} \in \mathscr{D}} \mathbb{E}\left[\left(-\alpha-\mathbf{a}^{T} \mathbf{r}\right)_{+}\right] \\
= & \sup _{\mathbf{w}^{T} \mathbf{r} \in S_{1}} \mathbb{E}\left[\left(-\alpha-\mathbf{w}^{T} \mathbf{r}\right)_{+}\right] \\
= & \sup _{\eta \in S_{2}} \mathbb{E}\left[(-\alpha-\eta)_{+}\right] \\
= & \sup _{\eta \sim\left(\mathbf{a}^{T} \boldsymbol{\mu}, \mathbf{a}^{T} \Sigma \mathbf{a}\right)} \mathbb{E}\left[(-\alpha-\eta)_{+}\right]
\end{array}
$$

$$
\begin{aligned}
& =\sup _{\eta \sim\left(\mathbf{w}^{T} \boldsymbol{\mu}, \mathbf{w}^{T} \Sigma \mathbf{w}\right)} \mathbb{E}\left[(-\alpha-\eta)_{+}\right] \\
& =\frac{1}{2}\left(\sqrt{\mathbf{w}^{T} \sum \mathbf{w}+\left(\boldsymbol{\mu}^{T} \mathbf{w}+\alpha\right)^{2}}-\left(\boldsymbol{\mu}^{T} \mathbf{w}+\alpha\right)\right) .
\end{aligned}
$$

The final equality follows from Lemma 3; this is the desired result. by

Hence, from Lemma 5, WCCVaR $(\mathbf{w})$ can be expressed

$$
\mathrm{WCCVaR}_{\beta}(\mathbf{w})
$$

$$
\begin{gathered}
=\min _{\alpha \in \mathbb{R}}\left\{\alpha+\frac{1}{2(1-\beta)}\left(\sqrt{\mathbf{w}^{T} \sum \mathbf{w}+\left(\boldsymbol{\mu}^{T} \mathbf{w}+\alpha\right)^{2}}\right.\right. \\
\left.\left.-\left(\boldsymbol{\mu}^{T} \mathbf{w}+\alpha\right)\right)\right\} .
\end{gathered}
$$

Clearly, the right side of the equation above is convex function in $\alpha$ and we can prove that $\mathrm{WCCVaR}_{\beta}(\mathbf{w})$ can be attained at

$$
\alpha^{*}=-\boldsymbol{\mu}^{T} \mathbf{w}+\frac{2 \beta-1}{2 \sqrt{\beta(1-\beta)}} \sqrt{\mathbf{w}^{T} \sum \mathbf{w}} ;
$$

that is,

$$
\operatorname{WCCVaR}_{\beta}(\mathbf{w})=-\boldsymbol{\mu}^{T} \mathbf{w}+\sqrt{\frac{\beta}{1-\beta}} \sqrt{\mathbf{w}^{T} \sum \mathbf{w}} .
$$

Then, robust mean-WCCVaR portfolio problem (RP1) can be expressed further as

$$
\begin{array}{ll}
\max _{\mathbf{w}} & \boldsymbol{\mu}^{T} \mathbf{w} \\
\text { s.t. } & -\boldsymbol{\mu}^{T} \mathbf{w}+\sqrt{\frac{\beta}{1-\beta}} \sqrt{\mathbf{w}^{T} \sum \mathbf{w}} \leq \tau, \\
& \mathbf{e}^{T} \mathbf{w}=1 .
\end{array}
$$

In the rest of this paper, we discuss mainly the solution of (RP2). To this end, let $a=\mathbf{e}^{T} \Sigma^{-1} \mathbf{e}, b=\boldsymbol{\mu}^{T} \Sigma^{-1} \mathbf{e}, c=\boldsymbol{\mu}^{T} \Sigma^{-1} \boldsymbol{\mu}$, $a_{0}=\left(a c-b^{2}\right) / a$, and $\beta_{0}=\sqrt{(1-\beta) / \beta}$. Then, the following result gives feasible conditions of problem (RP2).

Lemma 6. If $a_{0} \beta_{0}^{2}<1$ and

$$
\tau \geq \max \left\{\tau^{*}, 0\right\},
$$

then problem $(\mathrm{RP} 2)$ is feasible, where $\tau^{*}=\sqrt{1-a_{0} \beta_{0}^{2}} /\left(\beta_{0} \sqrt{a}\right)-$ $b / a$.

Proof. For any given $\beta \in(0,1)$, consider the problem

$$
\tau^{*}=\min _{\mathbf{w}}\left\{-\boldsymbol{\mu}^{T} \mathbf{w}+\sqrt{\frac{\beta}{1-\beta}} \sqrt{\mathbf{w}^{T} \Sigma \mathbf{w}} \mid \text { s.t. } \mathbf{e}^{T} \mathbf{w}=1\right\} .
$$


Let $v=\sqrt{\mathbf{w} \sum \mathbf{w}}$ and let $v$ be viewed as a new variable. Denote the optimal solution of problem (19) by $\widehat{\mathbf{w}}$; then $\widehat{\mathbf{w}}$ satisfies the first order condition:

$$
\begin{gathered}
-\boldsymbol{\mu}+\lambda^{\prime} \mathbf{e}+2 \lambda^{\prime \prime} \Sigma^{-1} \widehat{\mathbf{w}}=0, \\
2 \lambda^{\prime \prime} v=\sqrt{\frac{\beta}{1-\beta}}, \\
\mathbf{e}^{T} \widehat{\mathbf{w}}=1, \\
\widehat{\mathbf{w}}^{T} \sum \widehat{\mathbf{w}}=v^{2},
\end{gathered}
$$

where $\lambda^{\prime}, \lambda^{\prime \prime} \in \mathbb{R}$ are the Lagrange multipliers. It is not hard to obtain from the first and third equations of (20)

$$
\widehat{\mathbf{w}}=\frac{1}{2 \lambda^{\prime \prime}}\left(\Sigma^{-1} \boldsymbol{\mu}-\frac{b}{a} \Sigma^{-1} \mathbf{e}\right)+\frac{1}{a} \Sigma^{-1} \mathbf{e} .
$$

Substituting $\widehat{\mathbf{w}}$ in the fourth equation and combining the second equation of (20), then we can get the optimal solution of (19) when $a_{0} \beta_{0}^{2}<1$

$$
\widehat{\mathbf{w}}=\frac{\beta_{0}}{\sqrt{a\left(1-a_{0} \beta_{0}^{2}\right)}}\left(\Sigma^{-1} \boldsymbol{\mu}-\frac{b}{a} \Sigma^{-1} \mathbf{e}\right)+\frac{1}{a} \Sigma^{-1} \mathbf{e} .
$$

The optimal value

$$
\tau^{*}=\frac{\sqrt{1-a_{0} \beta_{0}^{2}}}{\beta_{0} \sqrt{a}}-\frac{b}{a} .
$$

If $\tau=\tau^{*}>0$, then $\widehat{\mathbf{w}}$ is unique solution of problem. This means that the feasible condition is $\tau \geq \max \left\{\tau^{*}, 0\right\}$. The proof is finished.

Lemma 6 means that the portfolio problem (RP2) is well defined if the investor chooses an appropriate risk tolerance parameter $\tau$. The following theorem gives the main results of the current paper.

Theorem 7. If $a_{0} \beta_{0}^{2}<1$ and $\tau>\max \left\{\tau^{*}, 0\right\}$, the optimal solution of the problem (RP2) can explicitly be expressed

$$
\mathbf{w}^{*}=f(\tau)\left(\Sigma^{-1} \boldsymbol{\mu}-\frac{b}{a} \Sigma^{-1} \mathbf{e}\right)+\frac{1}{a} \Sigma^{-1} \mathbf{e}
$$

where

$$
\begin{aligned}
& f(\tau) \\
& =\frac{a_{0} b \beta_{0}^{2}(\tau+b / a)+b \sqrt{a_{0} \beta_{0}^{2}(\tau+b / a)^{2}-\left(a_{0} / a\right)\left(1-a_{0} \beta_{0}^{2}\right)}}{a_{0}\left(1-a_{0} \beta_{0}^{2}\right)} .
\end{aligned}
$$

Proof. Let $v=\sqrt{\mathbf{w} \sum \mathbf{w}}$; the optimization problem (RP2) can be rewritten as

$$
\begin{array}{ll}
\max _{\mathbf{w}, v>0} & \mathbb{E}\left[\mathbf{r}^{T} \mathbf{w}\right] \\
\text { s.t. } & v-\beta_{0} \boldsymbol{\mu}^{T} \mathbf{w} \leq \beta_{0} \tau, \\
& \mathbf{e}^{T} \mathbf{w}=1, \\
& \mathbf{w}^{T} \Sigma \mathbf{w}=v^{2} .
\end{array}
$$

Let $\mathbf{w}^{*}$ be the optimal solution of problem (26). Then from KKT condition, $\mathbf{w}^{*}$ satisfies that

$$
\begin{gathered}
\left(1+\beta_{0} \lambda_{1}\right) \boldsymbol{\mu}-\lambda_{2} \mathbf{e}-2 \lambda_{3} \Sigma \mathbf{w}^{*}=0, \\
-\lambda_{1}+2 \lambda_{3} v=0 \\
\mathbf{e}^{T} \mathbf{w}^{*}=1, \\
\left(\mathbf{w}^{*}\right)^{T} \Sigma \mathbf{w}^{*}=v^{2} \\
v-\beta_{0} \boldsymbol{\mu}^{T} \mathbf{w}^{*}-\beta_{0} \tau=0
\end{gathered}
$$

where $\lambda_{1} \geq 0, \lambda_{2} \in \mathbb{R}$ and $\lambda_{3} \in \mathbb{R}$ are Lagrange multipliers. It follows from the first and third equations in (27) that

$$
\mathbf{w}^{*}=\frac{\left(1+\beta_{0} \lambda_{1}\right)}{2 \lambda_{3}}\left(\Sigma^{-1} \boldsymbol{\mu}-\frac{b}{a} \Sigma^{-1} \mathbf{e}\right)+\frac{1}{a} \Sigma^{-1} \mathbf{e} .
$$

Substituting $\mathbf{w}^{*}$ in the fourth and fifth equation in (27), we have

$$
\begin{gathered}
a_{0}\left[\frac{\left(1+\beta_{0} \lambda_{1}\right)}{2 \lambda_{3}}\right]^{2}+\frac{1}{a}=v^{2}, \\
a_{0} \beta_{0} \frac{\left(1+\beta_{0} \lambda_{1}\right)}{2 \lambda_{3}}=v-\beta_{0}\left(\tau+\frac{b}{a}\right) .
\end{gathered}
$$

Eliminating $v$ from (29), it follows that the quadratic equation with respect to $\left(1+\beta_{0} \lambda_{1}\right) / 2 \lambda_{3}$ is

$$
\begin{aligned}
& a_{0}\left(1-a_{0} \beta_{0}^{2}\right)\left(\frac{\left(1+\beta_{0} \lambda_{1}\right)}{2 \lambda_{3}}\right)^{2} \\
& \quad-2 a_{0} \beta_{0}^{2}\left(\tau+\frac{b}{a}\right)\left(\frac{\left(1+\beta_{0} \lambda_{1}\right)}{2 \lambda_{3}}\right)+\frac{1}{a}-\beta_{0}^{2}\left(\tau+\frac{b}{a}\right)^{2}=0 .
\end{aligned}
$$

Notice that $v>0$ and $\lambda_{1} \geq 0$; then from the second equation in (27), $\lambda_{3}>0$, this means that $\left(1+\beta_{0} \lambda_{1}\right) / 2 \lambda_{3}>0$. Solving directly the quadratic equation above in $\left(1+\beta_{0} \lambda_{1}\right) / 2 \lambda_{3}$, we have that

$$
\begin{aligned}
& \frac{\left(1+\beta_{0} \lambda_{1}\right)}{2 \lambda_{3}} \\
& \quad=\frac{a_{0} \beta_{0}^{2}(\tau+b / a)+\sqrt{a_{0} \beta_{0}^{2}(\tau+b / a)^{2}-\left(a_{0} / a\right)\left(1-a_{0} \beta_{0}^{2}\right)}}{a_{0}\left(1-a_{0} \beta_{0}^{2}\right)} \\
& >0 .
\end{aligned}
$$


Then the optimal solution of problem (RP2) can be obtained by substituting it in (27). This finishes the proof.

We need that condition $a_{0} \beta_{0}^{2}<1$ holds in Lemma 6 and Theorem 7 . This condition can in fact be easily attained. Notice that $\beta_{0}=\sqrt{(1-\beta) / \beta}$. Thus, for any input data $a_{0}$, in order to have $a_{0} \beta_{0}^{2}<1$, we only require that $\beta$ satisfies

$$
\beta>\frac{a_{0}}{1+a_{0}}
$$

If $\beta \in(1 / 2,1)$, the condition $a_{0} \beta_{0}^{2}<1$ can be satisfied while

$$
\beta>\max \left\{\frac{1}{2}, \frac{a_{0}}{1+a_{0}}\right\} .
$$

\section{Two-Fund Separation Theorem}

In our analysis of this section, we view $\mathbf{w}^{*}$ as a function of input parameter $\tau$; that is, we denote it by $\mathbf{w}^{*}=\mathbf{w}(\tau)$. Let

$$
S(\mathbf{w})=\left\{\mathbf{w}(\tau): \max \left\{\tau^{*}, 0\right\} \leq \tau<\infty\right\} .
$$

Then, set $S(\mathbf{w})$ is the solution space of problem (RP2). Now we are interested in the question that whether the solution of problem (RP2) satisfies the two-fund separation theorem or not.

Theorem 8. Let $\mathbf{w}\left(\tau_{1}\right), \mathbf{w}\left(\tau_{2}\right)\left(\tau_{1} \neq \tau_{2}\right)$ be two solutions of mean-WCCVaR model; that is, $\mathbf{w}\left(\tau_{1}\right), \mathbf{w}\left(\tau_{2}\right) \in S(\mathbf{w})$. Then for any $\tau \in\left[\max \left\{\tau^{*}, 0\right\}, \infty\right)$ and the corresponding solution $\mathbf{w}(\tau)$, there exists a real number $\theta$, such that

$$
\mathbf{w}(\tau)=\theta \mathbf{w}\left(\tau_{1}\right)+(1-\theta) \mathbf{w}\left(\tau_{2}\right)
$$

that is, the two-fund separation theorem holds.

Proof. Noting $f(\tau)$ in Theorem 7, the optimal solution of mean-WCCVaR portfolio problem (RP2) can be written in the simple form

$$
\mathbf{w}(\tau)=f(\tau) \mathbf{w}_{A}+(1-f(\tau)) \mathbf{w}_{\sigma},
$$

where $\mathbf{w}_{\sigma}=\left(\Sigma^{-1} \mathbf{e}\right) / a$ is the portfolio with the minimum variance and $\mathbf{w}_{A}=\left(\Sigma^{-1} \boldsymbol{\mu}\right) / b$. For given $\tau_{1}, \tau_{2} \in\left[\max \left\{\tau^{*}, 0\right\}, \infty\right)$, notice that $\tau_{1} \neq \tau_{2}$; it follows that $f\left(\tau_{1}\right) \neq f\left(\tau_{2}\right)$. Then for any $\tau \in\left[\max \left\{\tau^{*}, 0\right\}, \infty\right)$, let

$$
\theta=\frac{f(\tau)-f\left(\tau_{2}\right)}{f\left(\tau_{1}\right)-f\left(\tau_{2}\right)}
$$

we have

$$
f(\tau)=\theta f\left(\tau_{1}\right)+(1-\theta) f\left(\tau_{2}\right) .
$$

Thus

$$
\begin{aligned}
\mathbf{w}(\tau)= & f(\tau) \mathbf{w}_{A}+(1-f(\tau)) \mathbf{w}_{\sigma} \\
= & {\left[\theta f\left(\tau_{1}\right)+(1-\theta) f\left(\tau_{2}\right)\right] \mathbf{w}_{A} } \\
& +\left[1-\left[\theta f\left(\tau_{1}\right)+(1-\theta) f\left(\tau_{2}\right)\right]\right] \mathbf{w}_{\sigma} \\
= & \theta\left[f\left(\tau_{1}\right) \mathbf{w}_{A}+\left(1-f\left(\tau_{1}\right)\right) \mathbf{w}_{\sigma}\right] \\
& +(1-\theta)\left[f\left(\tau_{2}\right) \mathbf{w}_{A}+\left(1-f\left(\tau_{2}\right)\right) \mathbf{w}_{\sigma}\right] \\
= & \theta \mathbf{w}\left(\tau_{1}\right)+(1-\theta) \mathbf{w}\left(\tau_{2}\right) .
\end{aligned}
$$

This gets the desired conclusion.

\section{Efficient Frontier}

We discuss the efficient frontier of optimal solution of problem (RP2) and analyze the relationship of the efficient frontier between problem (RP2) and mean-variance (MV) model. For any given parameter $\tau$, clearly, the optimal solution $\mathbf{w}^{*}=$ $\mathbf{w}(\tau)$ and the expected return of portfolio are the function of $\tau$. Hence, we have

$R(\tau)$

$$
\begin{aligned}
= & \frac{a_{0} \beta_{0}^{2}(\tau+b / a)+\sqrt{a_{0} \beta_{0}^{2}(\tau+b / a)^{2}-\left(a_{0} / a\right)\left(1-a_{0} \beta_{0}^{2}\right)}}{\left(1-a_{0} \beta_{0}^{2}\right)} \\
& +\frac{b}{a} .
\end{aligned}
$$

Rearranging this equality, we have the quadratic equation in $R(\tau)$ and $\tau$

$$
\begin{aligned}
(1 & \left.-a_{0} \beta_{0}^{2}\right)^{2}\left(R(\tau)-\frac{b}{a}\right)^{2} \\
& -2 a_{0} \beta_{0}^{2}\left(1-a_{0} \beta_{0}^{2}\right)\left(R(\tau)-\frac{b}{a}\right)\left(\tau+\frac{b}{a}\right) \\
& +a_{0} \beta_{0}^{2}\left(a_{0} \beta_{0}^{2}-1\right)\left(\tau+\frac{b}{a}\right)^{2}+\frac{a_{0}}{a}\left(1-a_{0} \beta_{0}^{2}\right)=0 .
\end{aligned}
$$

The determinant of quadratic term coefficient can be expressed as

$$
\begin{aligned}
& \left|\begin{array}{cc}
\left(1-a_{0} \beta_{0}^{2}\right)^{2} & -a_{0} \beta_{0}^{2}\left(1-a_{0} \beta_{0}^{2}\right) \\
-a_{0} \beta_{0}^{2}\left(1-a_{0} \beta_{0}^{2}\right) & a_{0} \beta_{0}^{2}\left(a_{0} \beta_{0}^{2}-1\right)
\end{array}\right| \\
& =-a_{0} \beta_{0}^{2}\left(1-a_{0} \beta_{0}^{2}\right)^{2}<0 .
\end{aligned}
$$

This means from the theory of quadratic curve that the efficient frontier determined by (41) is a branch of the hyperbola and the portfolio at the efficient frontier has the maximum expected return for given $\tau$. The asymptotic line equation of efficient frontier is

$$
R(\tau)=\frac{1+\sqrt{a_{0} \beta_{0}^{2}}}{1-a_{0} \beta_{0}^{2}}\left(\sqrt{a_{0} \beta_{0}^{2}} \tau+\frac{b}{a}\right),
$$




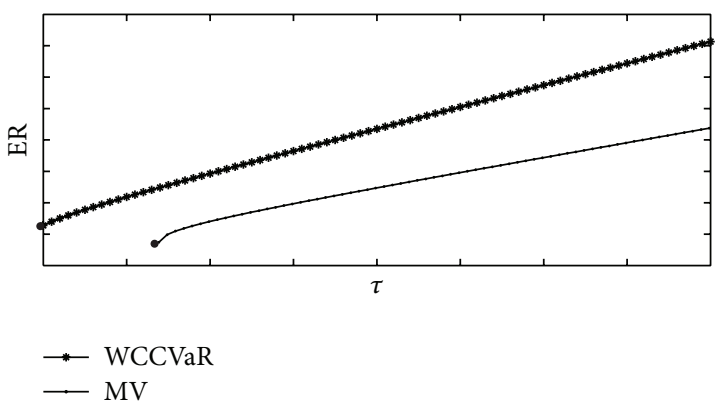

(a) $\beta_{0}>\beta_{1}$

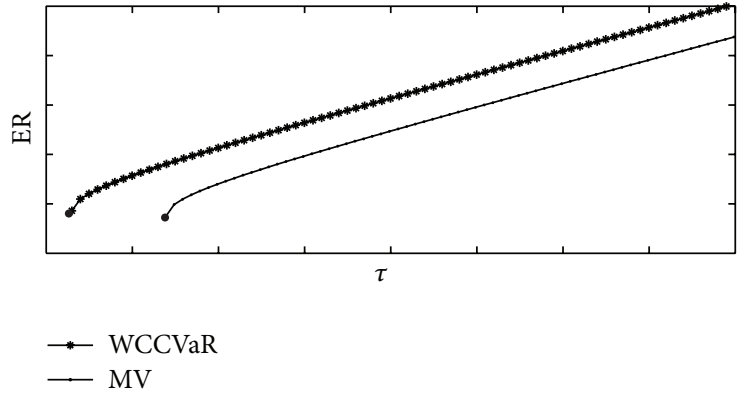

(b) $\beta_{0}=\beta_{1}$

FIGURE 1: The relationship of efficient frontiers of two models $\beta_{0} \geq \beta_{1}$.

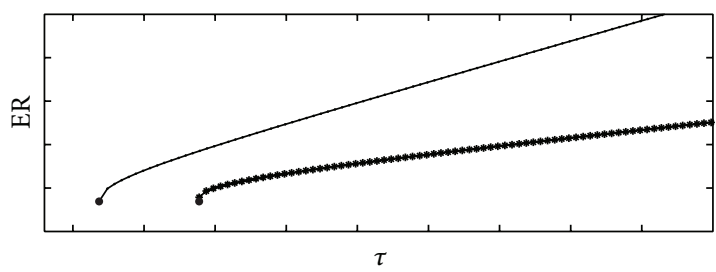

$\rightarrow$ WCCVaR
$-\mathrm{MV}$

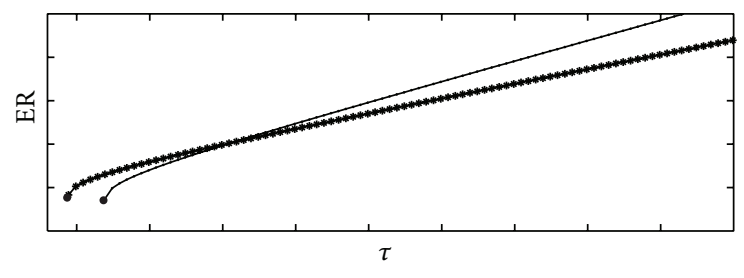

$\rightarrow$ WCCVaR

$-\mathrm{MV}$
(a) $\beta_{0}<\beta_{1}, \tau^{*}>\tau_{\mathrm{MV}}$
(b) $\beta_{0}<\beta_{1}, \tau^{*}<\tau_{\mathrm{MV}}$

FIGURE 2: The relationship of efficient frontiers of two models in the case of $\beta_{0}<\beta_{1}$.

the intercept at $R(\tau)$ axis is

$$
R_{b}=\frac{b}{a} \cdot \frac{1+\sqrt{a_{0} \beta_{0}^{2}}}{1-a_{0} \beta_{0}^{2}},
$$

and the center is $(-b / a, b / a)$. Hence, the location of the hyperbola is determined by sign of $b$.

Now, we will discuss a relationship of efficient frontiers between the mean-variance (MV) model and the proposed mean-WCCVaR model. To this end, we compare the meanWCCVaR model with the following MV model:

$$
\max \left\{E\left[\boldsymbol{\mu}^{T} \mathbf{w}\right]: \mathbf{w}^{T} \sum \mathbf{w} \leq \tau^{2}, \mathbf{e}^{T} \mathbf{w}=1\right\} .
$$

It is not hard to compute that the optimal solution of MV model is

$$
\begin{aligned}
\mathbf{w}_{\mathrm{MV}}^{*} & =\mathbf{w}_{\mathrm{MV}}(\tau) \\
& =\left(1-b \sqrt{\frac{\tau^{2}-1 / a}{a_{0}}}\right) \frac{\Sigma^{-1} \mathbf{e}}{a}+\sqrt{\frac{\tau^{2}-1 / a}{a_{0}}} \Sigma^{-1} \boldsymbol{\mu} .
\end{aligned}
$$

The corresponding expected value at the optimal solution is

$$
R_{\mathrm{MV}}(\tau)=\boldsymbol{\mu}^{T} \mathbf{w}_{\mathrm{MV}}^{*}=\frac{a c-b^{2}}{a} \sqrt{\frac{\tau^{2}-1 / a}{a_{0}}}+\frac{b}{a} .
$$

In $\left(\tau, R_{\mathrm{MV}}(\tau)\right)$ plane, function $R_{\mathrm{MV}}(\tau)$ plots the efficient frontier of MV:

$$
a \tau_{\mathrm{MV}}^{2}-\frac{a}{a_{0}}\left(R_{\mathrm{MV}}(\tau)-\frac{b}{a}\right)^{2}=1,
$$

whose slope of asymptotic line is $\sqrt{a_{0}}$. Let

$$
\frac{1+\sqrt{a_{0} \beta_{0}^{2}}}{1-a_{0} \beta_{0}^{2}} \sqrt{a_{0} \beta_{0}^{2}}=\sqrt{a_{0}} .
$$

Then,

$$
\beta_{0}=\frac{\sqrt{1+4\left(a_{0}+\sqrt{a_{0}}\right)}-1}{2\left(a_{0}+\sqrt{a_{0}}\right)}=: \beta_{1}
$$

If $\beta_{0} \geq \beta_{1}$, then for the case of $\tau^{*}<\tau_{\mathrm{MV}}$, two asymptotic lines are parallel and therefore efficient frontiers are not intersection; see Figure 1. If $\beta_{0}<\beta_{1}$, then the slope of asymptotic line of efficient frontier for mean-WCCVaR model is less than that of MV model and two efficient frontiers can be intersected when $\tau^{*}<\tau_{\mathrm{MV}}$; see Figure 2(b).

Generally speaking, it is more conservative for meanWCCVaR model than MV model. But the conservative performance can be improved by adjusted the confidence level $\beta$. For example, if $\beta$ is small, such that $\beta_{0}>\beta_{1}$, then meanWCCVaR has the higher expectation return at the same $\tau$; see Figure 1. This means that $\mathrm{ER} / \tau$ is also higher; that is, the expectation return of per unit WCCVaR risk (reflected by parameter $\tau$ ) is higher. The other case is that if we choose $\beta$, such that $\beta_{0}<\beta_{1}$, then mean-WCCVaR model can obtain still the large expected return at the same $\tau$ if $\tau$ is not large; see Figure 2. 


\section{An Extension with Risky-Free Asset}

We consider the portfolio with risky-free asset in this section and therefore the optimization problem can be expressed as

$$
\begin{array}{ll}
\max _{\mathbf{w}, w_{f}} & \mathbb{E}\left[\mathbf{r}^{T} \mathbf{w}+w_{f} r_{f}\right] \\
\text { s.t. } & -\boldsymbol{\mu}^{T} \mathbf{w}-w_{f} r_{f}+\sqrt{\frac{\beta}{1-\beta}} \sqrt{\mathbf{w}^{T} \sum \mathbf{w}} \leq \tau, \\
& \mathbf{e}^{T} \mathbf{w}+w_{f}=1 .
\end{array}
$$

Clearly, $\left(\mathbf{w}, w_{f}\right)=(\mathbf{0}, 1)$ is a strictly feasible solution of problem (RPF). Hence, for any $\tau>0$, problem (RPF) is always feasible. The following theorem gives the explicit solution of problem (RPF).

Theorem 9. If

$$
\beta_{0}^{2}\left[a_{0}+\frac{\left(b-a r_{f}\right)^{2}}{a}\right]<1
$$

then problem (RPF) has the optimal solution

$$
\begin{aligned}
\mathbf{w}^{*}= & \frac{\beta_{0}\left(\tau+r_{f}\right)}{\sqrt{a_{0}+\left(b-a r_{f}\right)^{2} / a}\left(1-\beta_{0} \sqrt{a_{0}+\left(b-a r_{f}\right)^{2} / a}\right)} \\
& \times\left(\Sigma^{-1} \boldsymbol{\mu}-r_{f} \Sigma^{-1} \mathbf{e}\right)
\end{aligned}
$$

where

$$
=1-\frac{\beta_{0}\left(\tau+r_{f}\right)\left(b-a r_{f}\right)}{\sqrt{a_{0}+\left(b-a r_{f}\right)^{2} / a}\left(1-\beta_{0} \sqrt{a_{0}+\left(b-a r_{f}\right)^{2} / a}\right)} .
$$

Proof. Let $v=\sqrt{\mathbf{w} \sum \mathbf{w}}$; similar to (RP2), optimization problem (RPF) can be rewritten as

$$
\begin{array}{ll}
\max _{\mathbf{w}, v>0} & \mathbb{E}\left[\mathbf{r}^{T} \mathbf{w}+r_{f} w_{f}\right] \\
\text { s.t. } & v-\beta_{0}\left(\boldsymbol{\mu}^{T} \mathbf{w}+r_{f} w_{f}\right) \leq \beta_{0} \tau, \\
& \mathbf{e}^{T} \mathbf{w}+r_{f} w_{f}=1, \\
& \mathbf{w}^{T} \Sigma \mathbf{w}=v^{2} .
\end{array}
$$

Then, from KKT condition, the optimal solution $\left(\mathbf{w}^{*}, w_{f}^{*}\right)$ must satisfy the first-order condition

$$
\begin{gathered}
\left(1+\beta_{0} \lambda_{f}^{\prime}\right) \boldsymbol{\mu}-\lambda_{f}^{\prime \prime} \mathbf{e}-2 \lambda_{f}^{\prime \prime \prime} \Sigma \mathbf{w}^{*}=0, \\
-\lambda_{f}^{\prime}+2 \lambda_{f}^{\prime \prime \prime} v=0, \\
\mathbf{e}^{T} \mathbf{w}^{*}=1-w_{f}^{*}, \\
\left(\mathbf{w}^{*}\right)^{T} \Sigma \mathbf{w}^{*}=v^{2}, \\
v-\beta_{0}\left(\boldsymbol{\mu}^{T} \mathbf{w}^{*}+r_{f} w_{f}^{*}\right)=\beta_{0} \tau, \\
\left(1+\lambda_{f}^{\prime} \beta_{0}\right) r_{f}-\lambda_{f}^{\prime \prime}=0,
\end{gathered}
$$

where $\lambda_{f}^{\prime} \geq 0, \lambda_{f}^{\prime \prime} \in \mathbb{R}$ and $\lambda_{f}^{\prime \prime \prime} \in \mathbb{R}$ are Lagrangian multipliers. From the first and third equalities, we have

$$
\mathbf{w}^{*}=\frac{\left(1+\beta_{0} \lambda_{f}^{\prime}\right)}{2 \lambda_{f}^{\prime \prime \prime}}\left(\Sigma^{-1} \boldsymbol{\mu}-\frac{b}{a} \Sigma^{-1} \mathbf{e}\right)+\frac{1-w_{f}}{a} \Sigma^{-1} \mathbf{e} .
$$

Substituting $\mathbf{w}^{*}$ into the fourth and fifth equalities in (55), then

$$
\begin{gathered}
a_{0}\left[\frac{\left(1+\beta_{0} \lambda_{f}^{\prime}\right)}{2 \lambda_{f}^{\prime \prime \prime}}\right]^{2}+\frac{1-w_{f}}{a}=v^{2}, \\
a_{0} \beta_{0} \frac{\left(1+\beta_{0} \lambda_{f}^{\prime}\right)}{2 \lambda_{f}^{\prime \prime \prime}}+\beta_{0}\left(\tau+\frac{b}{a}\left(1-w_{f}\right)+r_{f} w_{f}\right)=v .
\end{gathered}
$$

Combining (56), (57), and the third and sixth equations in system of equations (55), we have

$$
\frac{\left(1+\beta_{0} \lambda_{f}^{\prime}\right)}{2 \lambda_{f}^{\prime \prime \prime}}=\frac{1-w_{f}}{b-a r_{f}} .
$$

Eliminating $v$ and $1-w_{f}$ from (56), (57), and (58), we obtain a quadratic equation in $\left(1+\beta_{0} \lambda_{f}^{\prime}\right) / 2 \lambda_{f}^{\prime \prime \prime}$ :

$$
\begin{aligned}
& \left(a_{0}+\frac{\left(b-a r_{f}\right)^{2}}{a}\right)\left[1-\beta_{0}^{2}\left(a_{0}+\frac{\left(b-a r_{f}\right)^{2}}{a}\right)\right] \\
& \times\left(\frac{\left(1+\beta_{0} \lambda_{f}^{\prime}\right)}{2 \lambda_{f}^{\prime \prime \prime}}\right)^{2} \\
& -2 \beta_{0}^{2}\left(\tau+r_{f}\right)\left(a_{0}+\frac{\left(b-a r_{f}\right)^{2}}{a}\right)\left(\frac{\left(1+\beta_{0} \lambda_{f}^{\prime}\right)}{2 \lambda_{f}^{\prime \prime \prime}}\right) \\
& -\beta_{0}^{2}\left(\tau+r_{f}\right)^{2}=0 .
\end{aligned}
$$


TABLE 1: The mean and covariance matrix of returns for the chosen 9 indexes.

\begin{tabular}{cccccccccc}
\hline & II & CI1 & PI & UI & CI2 & HS & N225 & FTSE & DJIA \\
\hline $\boldsymbol{\mu}$ & 0.1813 & 0.1875 & 0.1924 & 0.2214 & 0.1636 & 0.1018 & -0.0096 & 0.0503 & 0.0849 \\
\hline & 0.2676 & 0.2785 & 0.3052 & 0.2909 & 0.2416 & 0.0992 & 0.0210 & 0.0330 & 0.0363 \\
& 0.2785 & 0.3109 & 0.3246 & 0.2859 & 0.2533 & 0.0983 & 0.0184 & 0.0330 & 0.0301 \\
& 0.3052 & 0.3246 & 0.3924 & 0.3177 & 0.3063 & 0.1147 & 0.0309 & 0.0314 & 0.0324 \\
& 0.2909 & 0.2859 & 0.3177 & 0.3611 & 0.2287 & 0.0964 & 0.0081 & 0.0315 & 0.0391 \\
& 0.2416 & 0.2533 & 0.3063 & 0.2287 & 0.3115 & 0.0881 & 0.0296 & 0.0370 & 0.0382 \\
& 0.0992 & 0.0983 & 0.1147 & 0.0964 & 0.0881 & 0.0950 & 0.0539 & 0.0332 & 0.0369 \\
& 0.0210 & 0.0184 & 0.0309 & 0.0081 & 0.0296 & 0.0539 & 0.0526 & 0.0248 & 0.0208 \\
& 0.0330 & 0.0330 & 0.0314 & 0.0315 & 0.0370 & 0.0332 & 0.0248 & 0.0262 & 0.0246 \\
& 0.0363 & 0.0301 & 0.0324 & 0.0391 & 0.0382 & 0.0369 & 0.0208 & 0.0246 & 0.0290 \\
\hline
\end{tabular}

TABLE 2: The out-of-sample returns statistics of portfolios obtained by WCCVaR, VaR, MV, and equally weighted strategy $(1 / N)$, where $1 / N$ is the equally weighted strategy. Std: standard deviation, tv: terminal value of wealth, and cv: coefficient of variation in this table.

\begin{tabular}{|c|c|c|c|c|c|c|}
\hline Model & Mean & Std & Max. & Min. & tv & $\mathrm{cV}$ \\
\hline WCCVaR & 0.0377 & 0.0131 & 0.0707 & -0.0002 & 1.0269 & $1.26 \%$ \\
\hline VaR & 0.0037 & 0.1169 & 0.2342 & -0.1987 & 0.8141 & $11.65 \%$ \\
\hline $1 / N$ & -0.1238 & 0.0759 & 0.0127 & -0.2485 & 0.8369 & $8.66 \%$ \\
\hline MV & 0.0046 & 0.0986 & 0.0457 & -0.1254 & 0.8433 & $8.53 \%$ \\
\hline
\end{tabular}

Solving directly this equation, we have that

$$
\begin{aligned}
& \frac{\left(1+\beta_{0} \lambda_{f}^{\prime}\right)}{2 \lambda_{f}^{\prime \prime \prime}} \\
& =\frac{\beta_{0}\left(\tau+r_{f}\right)}{\sqrt{a_{0}+\left(b-a r_{f}\right)^{2} / a}\left(1-\beta_{0} \sqrt{a_{0}+\left(b-a r_{f}\right)^{2} / a}\right)}
\end{aligned}
$$

And further, we can obtain $w_{f}$ from (58). Then, substituting $\left(1+\beta_{0} \lambda_{f}^{\prime}\right) / 2 \lambda_{f}^{\prime \prime \prime}$ and $w_{f}$ in (55), we get the results of this theorem.

It is not hard to compute the relationship between the expectation return $E_{f}$ of portfolio with risky-free asset and the parameter $\tau$ :

$$
\begin{aligned}
E_{f}(\tau)= & \frac{\beta_{0}\left(c-a r_{f}^{2}-2 b r_{f}\right)}{A} \tau \\
& +\left[\frac{\beta_{0}\left(c-a r_{f}^{2}-2 b r_{f}\right)}{A}+1\right] r_{f},
\end{aligned}
$$

where $A=\sqrt{a_{0}+\left(b-a r_{f}\right)^{2} / a}\left(1-\beta_{0} \sqrt{a_{0}+\left(b-a r_{f}\right)^{2} / a}\right)$ is independent of $\tau$.

\section{Numerical Results}

We take five domestic risky assets, industrial index (II), commercial index (CI1), properties index (PI), utilities index (UI), and composite index (CI2), and four overseas risky assets,
Hengsheng index (HS), Tokyo Nikkei-225 Index (N225), FTSE Index, and Dow-Jones industrial average index (DJIA). The time interval is from January 2, 1995, to December 31, 2012. The returns and covariance matrix of all risky assets can be found in Table 1. For simplicity, we take the risky-free annual interest rate is $r_{f}=3 \%$.

The rolling procedure is used to test the proposed model as follows.

(i) We estimate first the parameters $\boldsymbol{\mu}$ and $\Sigma$ using the 15year data from January 2, 1995, to December 31, 2009, and test the out-of-sample performance at the whole 2010 year.

(ii) And then we further estimate the parameters $\boldsymbol{\mu}$ and $\Sigma$ using the next 15-year data from January 2, 1996, to December 31, 2010, and test the out-of-sample performance at the whole 2011 year.

(iii) We finally estimate the parameters $\boldsymbol{\mu}$ and $\Sigma$ using the next 15-year data from January 2, 1997, to December 31,2011 , and test the out-of-sample performance at the whole 2012 year.

In our numerical reports, we compare our WCCVaR model with the classical VaR model under normal distribution assumption, equally weighted strategy [17], and MV model. Table 2 gives the results of three models with $\beta=95 \%$ and $\tau=0.05$. The following observations can be found from Table 2 .

(1) The standard deviation of portfolio obtained by WCCVaR model is clearly less than that of VaR model and equally weighted strategy [17]; moreover, the expected return of portfolio obtained by WCCVaR model is greater than that of VaR model and equally weighted strategy [17]. This means that the proposed 
WCCVaR model has the better performance ( such as Sharpe ratio).

(2) The real wealth of WCCVaR is at least 0.9998 that is very close to the initial wealth one and the coefficient of variation is only $1.26 \%$ which is far less than the coefficient of variation of VaR model and equally weighted strategy. The stable performance of WCC$\mathrm{VaR}$ is obvious.

(3) At the beginning period, within the 300 trade dates, the accumulation wealth of VaR and MV model is better than that of WCCVaR, but, after about 300 trade dates, the accumulation wealth of VaR and MV falls rapidly while WCCVaR still holds the stable wealth. We find that the 300th trade date is about corresponding to the first two months of 2011; the real market at that time is a bear market. This is the main reason that the accumulation wealth of $\mathrm{VaR}$, equally weighted strategy and MV falls rapidly.

\section{Conclusions}

We discuss the worst-case CVaR risk measure without the distribution assumption and consider an application in robust portfolio selection problem. The explicit solution is obtained and two-fund separation theorem is proved for the solutions. The theoretical comparison with classical meanvariance model is first discussed by the efficient frontier and the numerical comparison with $\mathrm{VaR}, \mathrm{MV}$ model and equally weighted strategy using domestic and overseas assets. The numerical results indicate that the proposed WCCVaR has the better expected return and smaller standard deviation than VaR, MV model and equally weighted strategy and therefore can obtain the better performance, such as Sharpe ratio.

\section{Conflict of Interests}

The authors declare that there is no conflict of interests regarding the publication of this paper.

\section{Acknowledgments}

This work is supported by National Natural Science Foundations of China (71371090), Science Foundation of Ministry of Education of China (13YJCZH160), Natural Science Foundation of Jiangxi Province of China (20114BAB211008), and Jiangxi University of Finance and Economics Support Program Funds for Outstanding Youths.

\section{References}

[1] P. Artzner, F. Delbaen, and J.-M. Eber, "Coherent measures of risk," Mathematical Finance, vol. 9, no. 3, pp. 203-228, 1999.

[2] R. T. Rockafellar and S. Uryasev, "Optimization of conditional value-at-risk," The Journal of Risk, vol. 2, no. 3, pp. 21-41, 2000.

[3] D. Goldfarb and G. Iyengar, "Robust portfolio selection problems," Mathematics of Operations Research, vol. 28, no. 1, pp. 138, 2003.
[4] O. L. V. Costa and A. C. Paiva, "Robust portfolio selection using linear-matrix inequalities," Journal of Economic Dynamics \& Control, vol. 26, no. 6, pp. 889-909, 2002.

[5] B. V. Halldórsson and R. H. Tütüncü, "An interior-point method for a class of saddle-point problems," Journal of Optimization Theory and Applications, vol. 116, no. 3, pp. 559-590, 2003.

[6] R. H. Tütüncü and M. Koenig, "Robust asset allocation," Annals of Operations Research, vol. 132, no. 1-4, pp. 157-187, 2004.

[7] Z. Lu, "Robust portfolio selection based on a joint ellipsoidal uncertainty set," Optimization Methods and Software, vol. 26, no. 1, pp. 89-104, 2011.

[8] A.-F. Ling and C.-X. Xu, "Robust portfolio selection involving options under a'marginal + joint" ellipsoidal uncertainty set," Journal of Computational and Applied Mathematics, vol. 236, no. 14, pp. 3373-3393, 2012.

[9] L. El Ghaoui, M. Oks, and F. Oustry, "Worst-case value-atrisk and robust portfolio optimization: a conic programming approach," Operations Research, vol. 51, no. 4, pp. 543-556, 2003.

[10] S. Zhu and M. Fukushima, "Worst-case conditional value-atrisk with application to robust portfolio management," Operations Research, vol. 57, no. 5, pp. 1155-1168, 2009.

[11] S. Zhu, D. Li, and S. Wang, "Robust portfolio selection under downside risk measures," Quantitative Finance, vol. 9, no. 7, pp. 869-885, 2009.

[12] D. Huang, S. Zhu, F. J. Fabozzi, and M. Fukushima, "Portfolio selection with uncertain exit time: a robust CVaR approach," Journal of Economic Dynamics and Control, vol. 32, no. 2, pp. 594-623, 2008.

[13] F. J. Fabozzi, D. Huang, and G. Zhou, "Robust portfolios: contributions from operations research and finance," Annals of Operations Research, vol. 176, no. 1, pp. 191-220, 2010.

[14] L. Chen, S. He, and S. Zhang, "Tight bounds for some risk measures, with applications to robust portfolio selection," Operations Research, vol. 59, no. 4, pp. 847-865, 2011.

[15] M. S. Bazaraa, H. D. Sherali, and C. M. Shetty, Nonlinear Programming: Theory and Algorithms, Second Edition, John Wiley \& Sons, New York, NY, USA, 2nd edition, 1993.

[16] I. Popescu, "Robust mean-covariance solutions for stochastic optimization," Operations Research, vol. 55, no. 1, pp. 98-112, 2007.

[17] V. DeMiguel, L. Garlappi, and R. Uppal, "Optimal versus naive diversification: how inefficient is the $1 / \mathrm{N}$ portfolio strategy?" The Review of Financial Studies, vol. 22, no. 5, pp. 1915-1953, 2009. 


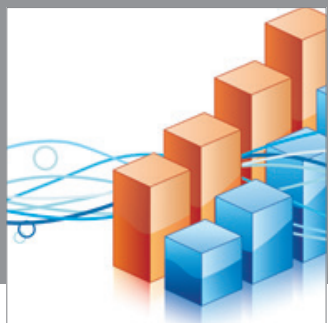

Advances in

Operations Research

mansans

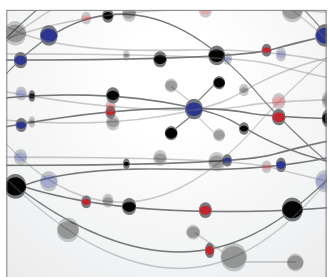

The Scientific World Journal
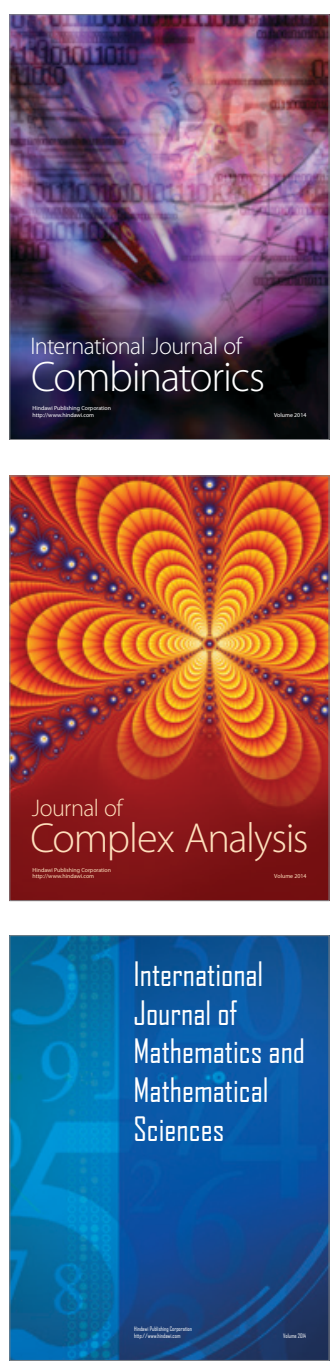
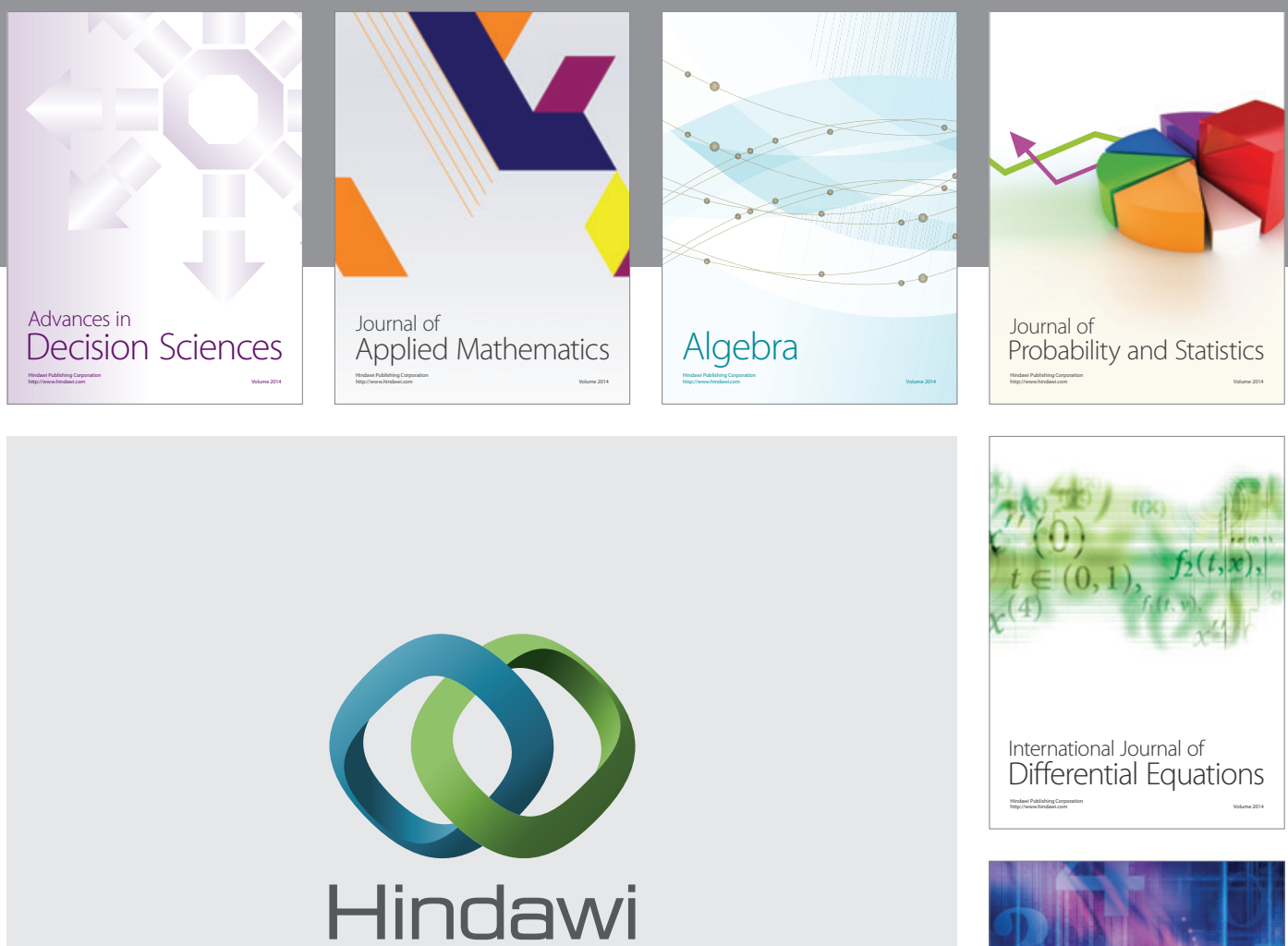

Submit your manuscripts at http://www.hindawi.com
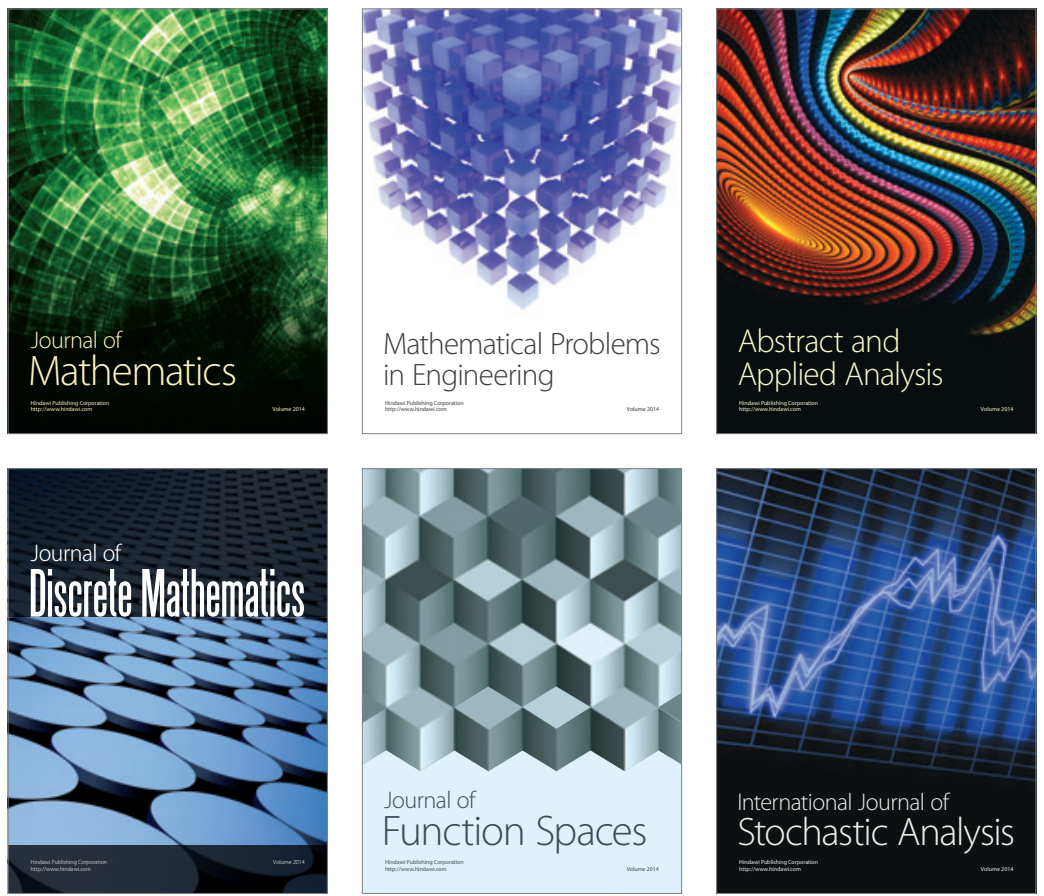

Journal of

Function Spaces

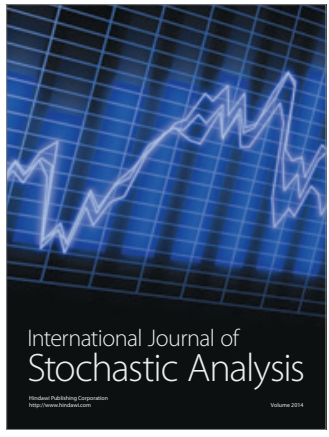

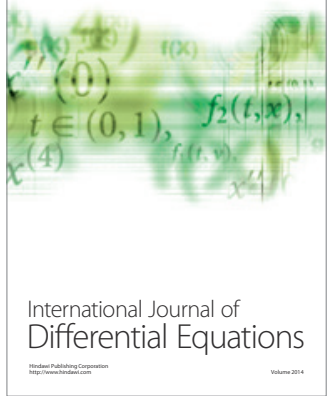
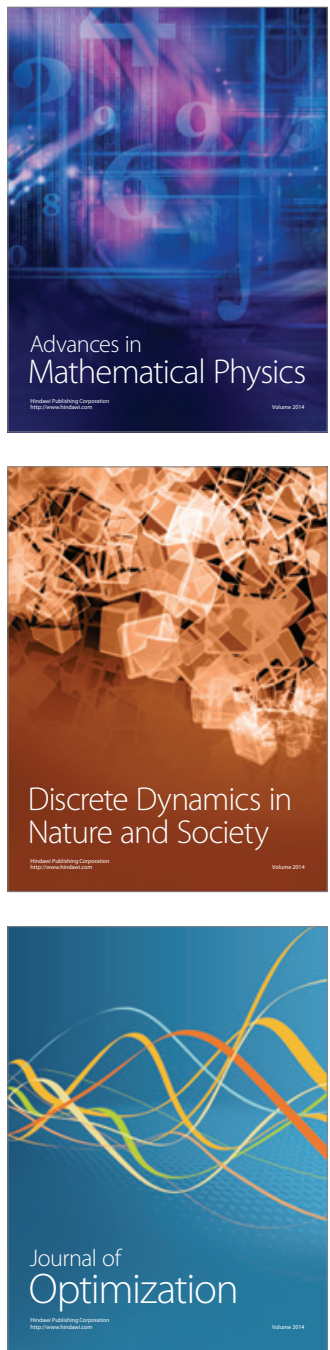